\title{
Convoluted Fractional $C$-Semigroups and Fractional Abstract Cauchy Problems
}

\author{
Zhan-Dong Mei, ${ }^{1,2}$ Ji-Gen Peng, ${ }^{1}$ and Jing-Huai Gao ${ }^{2}$ \\ ${ }^{1}$ School of Mathematics and Statistics, Xi'an Jiaotong University, Xi'an 710049, China \\ ${ }^{2}$ Institute of Wave and Information, School of Electronics and Information Engineering, Xi'an Jiaotong University, Xi'an 710049, China \\ Correspondence should be addressed to Zhan-Dong Mei; zhdmei@mail.xjtu.edu.cn
}

Received 30 September 2013; Accepted 12 December 2013; Published 12 January 2014

Academic Editor: Juan J. Trujillo

Copyright (c) 2014 Zhan-Dong Mei et al. This is an open access article distributed under the Creative Commons Attribution License, which permits unrestricted use, distribution, and reproduction in any medium, provided the original work is properly cited.

We present the notion of convoluted fractional $C$-semigroup, which is the generalization of convoluted $C$-semigroup in the Banach space setting. We present two equivalent functional equations associated with convoluted fractional $C$-semigroup. Moreover, the well-posedness of the corresponding fractional abstract Cauchy problems is studied.

\section{Introduction}

Let $X$ be a Banach space and let $C \in L(X)(L(X)$ denotes the space of bounded linear operators from $X$ to itself) be injective. Let $\Theta(t):=\int_{0}^{t} K(s) d s$, where $K(\cdot)$ is locally integrable on $[0,+\infty)$. A strongly continuous operator family $\{S(t)\}_{t \geq 0}$ is called a $K$-convoluted $C$-semigroup if $S(0)=0, S(t) C=$ $C S(t), t \geq 0$, and there holds

$$
\begin{array}{r}
S(t) S(s) x=\left[\int_{0}^{t+s}-\int_{0}^{t}-\int_{0}^{s}\right] K(t+s-r) S(r) C x d r, \\
x \in X, t, s \geq 0 .
\end{array}
$$

If, in addition, $S(t) x=0, t \geq 0$, implies $x=0,\{S(t)\}_{t \geq 0}$ is called a nondegenerate $K$-convoluted $C$-semigroup. For more details, we refer to Kostić [1]. Obviously, $K$-convoluted $C$ semigroups are a generalization of classical $C_{0}$-semigroups. The extension of Widder's representation theorem by Arendt [2] stimulated the development of the theory of $\alpha$-times integrated semigroups, which is the special case that $K(t)=$ $t^{\alpha-1} / \Gamma(\alpha), C=I$ (see [3-6] for $\alpha$ being an integer and [79] for $\alpha$ being noninteger). Li and Shaw [10-12] introduced exponentially bounded $n$-times integrated $C$-semigroups which are the special case, where $K(t)=t^{n-1} /(n-1)$ !, and they studied their connection with the associated abstract Cauchy problem. Kuo and Shaw [13] were concerned with the case $K(t)=t^{\alpha-1} / \Gamma(\alpha)$ with $\alpha$ being noninteger and they called it $\alpha$-times integrated $C$-semigroups. Convoluted semigroups, which are the special case that $C=I$, were introduced by Cioranescu and Lumer [14-16]. It is a generalization of integrated semigroups. Moreover, Kunstmann [17] showed that there exists a global convoluted semigroup whose generator $A$ is not stationary dense and therefore it cannot be the generator of a local integrated semigroup. After that, Melnikova and Filinkov [18], Keyantuo et al. [19], and Kostić and Pilipović [20] systematically studied the properties of convoluted semigroups and related them to associated abstract Cauchy problems.

In [1], Kostić presented the notion $K$-convoluted $C$ semigroup and found a sufficient condition for a nondegenerate strongly continuous linear operator family $\{S(t)\}_{t \geq 0}$ to be a $K$-Convoluted $C$-semigroup; that is, $S(t) A \subset A S(t)$, $C S(t)=S(t) C, t \geq 0, \int_{0}^{t} S(s) x d s \in D(A), x \in X$, and $S(t) x=$ $A \int_{0}^{t} S(s) x d s+\Theta(t) C x$. Moreover, Kostić showed that if the sufficient condition holds, the abstract Cauchy problem

$$
\begin{gathered}
u^{\prime}(t)=A u(t)+\Theta(t) C x, \\
u(0)=0,
\end{gathered}
$$


is well-posed. In Proposition 2.3 of [3], it was proved that if the following abstract Cauchy problem

$$
\begin{gathered}
u^{\prime}(t)=A u(t)+\frac{t^{\alpha}}{\Gamma(\alpha+1)} C x, \\
u(0)=0,
\end{gathered}
$$

is well-posed, then the above sufficient conditions hold. Motivated by such facts, Kostić gave in [1] the definition of $K$ convoluted semigroup by the above sufficient conditions and proved the equivalence of the two definitions. Later, Kostic and Pilipović [21] gave the definition of $K$-convoluted $C$ semigroups by using the sufficient condition.

Observe that the abstract Cauchy problems related to the above "semigroups" are of integral order. Recently, fractional differential equations have received increasing attention because the behavior of many physical systems, such as fluid flows, electrical networks, viscoelasticity, chemical physics, electron-analytical chemistry, biology, and control theory, can be properly described by using the fractional order system theory and so forth (see [22-26]). Fractional derivatives appear in the theory of fractional differential equations; they describe the property of memory and heredity of materials, and it is the major advantage of fractional derivatives compared with integer order derivatives.

In [27], Bajlekova developed the notion of solution operator to study the well-posedness of fractional differential equations. da Prato and Iannelli [28] introduced the concept of resolvent families, which can be regarded as an extension of $C_{0}$-semigroups, to study a class of integrodifferential equations. After that, the theory of resolvent families was developed rapidly to investigate the abstract Volterra equation:

$$
u(t)=f(t)+\int_{0}^{t} a(t-s) A u(s) d s,
$$

where $a \in L_{\text {loc }}^{1}(R+)$ (see [29-31]). Recently, Chen and Li [32] gave the family of functional equations, using resolvent operator function to equivalently describe resolvent families. Motivated by this, Kexue and Jigen [33, 34], Li et al. [35], and Mei et al. [36] studied different types of fractional resolvents describing the functional equation and applied them to fractional differential equations. Lizama and Poblete [37] studied the functional equation associated with $(a, k)$-regularized resolvents. However, the above functional equations are not expressed in terms of the sum of time variables: $s+t$. This is very important in concrete applications of the functional equation, just like $C_{0}$-semigroups, integrated semigroups, integrated $C$-semigroups, and $K$-convoluted $C$-semigroups (see (1)). In [38], Peng and Li derived a new characterization of $\alpha$-order semigroup $(0<\alpha<1)$ as follows:

$$
\begin{gathered}
\int_{0}^{t+s} \frac{T(\tau)}{(t+s-\tau)^{\alpha}} d \tau-\int_{0}^{t} \frac{T(\tau)}{(t+s-\tau)^{\alpha}} d \tau-\int_{0}^{s} \frac{T(\tau)}{(t+s-\tau)^{\alpha}} d \tau \\
\quad=\alpha \int_{0}^{t} \int_{0}^{s} \frac{T\left(r_{1}\right) T\left(r_{2}\right)}{\left(t+s-r_{1}-r_{2}\right)^{1+\alpha}} d r_{1} d r_{2}, \quad t, s \geq 0,
\end{gathered}
$$

which is proved to be equivalent to fractional resolvents introduced in [32]. Motivated by this, Mei et al. [39] presented a characterization as follows:

$$
\begin{array}{r}
\Gamma(1-\alpha) T(t+s)=\alpha \int_{0}^{t} \int_{0}^{s} \frac{T\left(r_{1}\right) T\left(r_{2}\right)}{\left(t+s-r_{1}-r_{2}\right)^{1+\alpha}} d r_{1} d r_{2}, \\
t, s>0,0<\alpha<1,
\end{array}
$$

which is proved to be equivalent to fractional resolvent presented by Kexue and Jigen [34]. One of the tasks of the present paper is to find a characterization of $K$-convoluted $\alpha$-order $C$-semigroup (see Section 2 for the definition) in terms of $t+s$.

In this paper, we consider the following fractional abstract Cauchy problem (FACP):

$$
\begin{gathered}
{ }^{C}{ }_{D_{t}^{\alpha} u(t)}^{\alpha}=A u(t)+\Theta(t) C x, \\
u(0)=0,
\end{gathered}
$$

where $0<\alpha<1, x \in X$, and ${ }^{C} D_{t}^{\alpha}$ is the modified Caputo fractional derivative defined by operator

$$
{ }^{C} D_{t}^{\alpha} u(t)=\frac{1}{\Gamma(1-\alpha)} \frac{d}{d t} \int_{0}^{t}(t-\sigma)^{-\alpha}(u(\sigma)-u(0)) d \sigma .
$$

Observe that fractional integral of $u(t)$ is defined by

$$
J_{t}^{\alpha} u(t)=\frac{1}{\Gamma(\alpha)} \int_{0}^{t}(t-s)^{\alpha-1} u(s) d s .
$$

Then it is easy to show that, for $u \in C^{1}([0, \infty), X),{ }^{C} D_{t}^{\alpha} u(t)$ can be calculated by

$$
{ }^{C} D_{t}^{\alpha} u(t)=J_{t}^{1-\alpha} u^{\prime}(s) d s,
$$

which is just the original definition of Caputo fractional derivative (see, e.g., [26]).

We plan to present in Section 2 the notion of $K$ convoluted fractional $C$-semigroups, which is a generalization of the notions of $K$-convoluted semigroups [20] and $K$ convoluted C-semigroups [21]. Moreover, some of their properties are studied. Section 3 is to give two equivalent descriptions of $K$-convoluted $C$-semigroup. The final section concerns the study of the well-posedness of the fractional abstract Cauchy problem.

Throughout this paper, $\alpha \in(0,1)$, without any additional statement.

\section{Convoluted Fractional $C$-Semigroup}

In this section, we will introduce the notion of $K$-convoluted $\alpha$-order $C$-semigroup and study some of its properties. The following definition is stimulated by the definitions of $K$ convoluted semigroup [20] and $K$-convoluted $C$-semigroup [21]. 
Definition 1. Let $A$ be a closed linear operator on a Banach space $X$. If there exists a strongly continuous operator family $\left\{S_{K}(t)\right\}_{t \geq 0}$ such that $S_{K}(t) C=C S_{K}(t), S_{K}(t) A \subset A S_{K}(t)$, $J_{t}^{\alpha} S_{K}(t) x \in D(A), x \in X, t \geq 0$, and

$$
A J_{t}^{\alpha} S_{K}(t) x=S_{K}(t) x-\Theta(t) C x, \quad x \in X,
$$

then $\left\{S_{K}(t)\right\}_{t \geq 0}$ is called a $K$-convoluted $\alpha$-order $C$-semigroup generated by $A$, and $A$ is called the generator of $\left\{S_{K}(t)\right\}_{t \geq 0}$. In the special case $C=I,\left\{S_{K}(t)\right\}_{t \geq 0}$ is called $K$-convoluted $\alpha$ order semigroup. One says that $\left\{S_{K}(t)\right\}_{t \geq 0}$ is an exponentially bounded $K$-convoluted $\alpha$-order $C$-semigroup generated by $A$ if, additionally, there exist $M>0$ and $\omega \in R$ such that $\left\|S_{K}(t)\right\| \leq M e^{\omega t}, t \geq 0$.

Remark 2. We note that $K$-convoluted $\alpha$-order $C$-semigroup is an $(a, k)$ regularized $C$-resolvent family $\left(a(t)=t^{\alpha-1} / \Gamma(\alpha)\right.$ and $\left.k(t)=\int_{0}^{t} K(s) d s\right)$, introduced by Kostić [40]. We are concerned with this case because it is closely related to fractional differential equation. There are three reasons why we use such concept: (1) Kostić and Pilipović called the special case $\alpha=1$ and $C=I$ to be $K$-convoluted semigroup [20] and $\alpha=2$ to be $K$-convoluted $C$-cosine function [21]; (2) Peng and Li called $\left(t^{\alpha-1} / \Gamma(\alpha), 1\right)$-regularized resolvent $\alpha$-order semigroup $(\alpha \epsilon$ $(0,1))$ [38]; (3) “ $K$-convoluted $\alpha$-order $C$-semigroup" should be " $\left(t^{\alpha-1} / \Gamma(\alpha), \int_{0}^{t} K(s) d s\right)$-regularized $C$-resolvent," but the term “ $\left(t^{\alpha-1} / \Gamma(\alpha), \int_{0}^{t} K(s) d s\right)$-regularized $C$-resolvent” is too complex.

It can be checked directly that if $A$ generates a $K$ convoluted $\alpha$-order $C$-semigroup $\left\{S_{K}(t)\right\}_{t \geq 0}$, then $S_{K}(t) x \in$ $\overline{D(A)}, t \geq 0, x \in X$. If $K \neq 0$ in $L_{\text {loc }}^{1}([0, \infty))$, then it is clear that the $K$-convoluted $\alpha$-order $C$-semigroup $\left\{S_{K}(t)\right\}_{t \geq 0}$ is nondegenerate (let $S_{K}(t) x=0, t>0$; (11) implies that $\Theta(t) C x=0$; thereby $x=0)$.

Lemma 3. Assume that $\left\{S_{K}(t)\right\}_{t \geq 0}$ is a nondegenerate $K$ convoluted $\alpha$-order $C$-semigroup generated by $A$ on Banach space $X$. Then $C A \subset A C$.

Proof. Let $x \in D(A)$. By Definition 1, it follows that $J_{t}^{\alpha} S_{K}(t) C x \in D(A), t \geq 0$, and

$$
A J_{t}^{\alpha} S_{K}(t) C x=S_{K}(t) C x-\Theta(t) C^{2} x
$$

The combination of the closedness of $A$ and (11) implies that

$$
J_{t}^{\alpha} A S_{K}(t) x=S_{K}(t) x-\Theta(t) C x,
$$

which gives that

$$
C J_{t}^{\alpha} A S_{K}(t) x=C S_{K}(t) x-\Theta(t) C^{2} x .
$$

The two equalities (12) and (14) indicate that $A J_{t}^{\alpha} S_{K}(t) C x=$ $C J_{t}^{\alpha} A S_{K}(t) x$. Hence,

$$
J_{t}^{1-\alpha} A J_{t}^{\alpha} S_{K}(t) C x=J_{t}^{1-\alpha} J_{t}^{\alpha} S_{K}(t) C A x=\int_{0}^{t} S_{K}(s) C A x d s,
$$

Since $A$ is closed, it follows that

$$
A \int_{0}^{t} S_{K}(s) C x d s=A J_{t}^{1-\alpha} J_{t}^{\alpha} S_{K}(t) C x=\int_{0}^{t} S_{K}(s) C A x d s,
$$

Using the closedness of $A$ again, we obtain that $S_{K}(t) C A x=$ $A S_{K}(t) C x=S_{K}(t) A C x, t \geq 0$. Since $\{S(t)\}_{t \geq 0}$ is nondegenerate, it follows that $C A x=A C x, x \in D(A)$. The proof is complete.

Theorem 4. Assume that $\left\{S_{K}(t)\right\}_{t \geq 0}$ is a nondegenerate $K$ convoluted $\alpha$-order $C$-semigroup generated by $A$ on Banach space $X$. Define the operator B by

$$
\begin{gathered}
x \in D(B), \\
B x=y \Longleftrightarrow J_{t}^{\alpha} S_{K}(t) y=S_{K}(t) x-\Theta(t) C x, \quad \forall t \geq 0 .
\end{gathered}
$$

Then

(i) $B$ is single-valued;

(ii) $B$ is closed;

(iii) $B=C^{-1} A C$.

Proof. We first prove that the nondegeneracy implies that $B$ is single-valued. Indeed, if, for any $t \geq 0, y, z \in X$ satisfy, respectively,

$$
\begin{aligned}
& S_{K}(t) x-\Theta(t) C x=J_{t}^{\alpha} S_{K}(s) y d s \\
& S_{K}(t) x-\Theta(t) C x=J_{t}^{\alpha} S_{K}(s) z d s,
\end{aligned}
$$

then

$$
J_{t}^{\alpha} S_{K}(s) y d s=J_{t}^{\alpha} S_{K}(s) z d s .
$$

By Titchmarsh's theorem, it follows that $S_{K}(s) y=S_{K}(s) z, s \geq$ 0 . The nondegeneracy implies that $y=z$; thereby $A$ is single valued. Hence we have $A x=y$.

Let the sequence $\left\{x_{n}\right\} \subset D(B)$ satisfy $x_{n} \rightarrow x$ and $B x_{n} \rightarrow$ $y$ as $n \rightarrow \infty$. Then we have

$$
J_{t}^{\alpha} S_{K}(t) A x_{n}=S_{K}(t) x_{n}-\Theta(t) C x_{n}, \quad \forall t \geq 0 .
$$

Letting $n \rightarrow \infty$, (43) gives that

$$
J_{t}^{\alpha} S_{K}(t) y=S_{K}(t) x-\Theta(t) C x, \quad \forall t \geq 0,
$$

which implies that $x \in D(B)$ and $y=B x$. Hence $B$ is closed.

If $x \in D(B)$, then $S_{K}(t) x-\Theta(t) C x=J_{t}^{\alpha} S_{K}(t) B x, t \geq 0$. The combination of (11) and (17) implies that

$$
A J_{t}^{\alpha} S_{K}(t) x=J_{t}^{\alpha} S_{K}(t) B x, \quad t \geq 0 .
$$

Similar to the proof of Lemma 3, using the closedness of $A$, we obtain

$$
A S_{K}(t) x=S_{K}(t) B x
$$


that is, $A\left(\Theta(t) C x+J_{t}^{\alpha} S_{K}(t) B x\right)=S_{K}(t) B x$. Since $\Theta \neq 0$ and $J_{t}^{\alpha} S_{K}(t) B x \in D(A)$, we obtain that $C x \in D(A)$. Combining Lemma 3, Definition 1, and (23), it follows that $S_{K}(t) C B x=$ $C S_{K}(t) B x=C A S_{K}(t) x=A C S_{K}(t) x=A S_{K}(t) C x=$ $S_{K}(t) A C x$. Since $\{S(t)\}_{t \geq 0}$ is nondegenerate, it follows that $C B x=A C x$; that is, $B x=C^{-1} A C x$. So $B \subset C^{-1} A C$. Conversely, let $x \in D\left(C^{-1} A C\right)$; that is, $C x \in D(A)$ and $A C x \in R(C)$. By Definition $1, J_{t}^{\alpha} S_{K}(t) C x \in D(A)$ and

$$
A J_{t}^{\alpha} S_{K}(t) C x=S_{K}(t) C x-\Theta(t) C^{2} x,
$$

which implies that

$$
C^{-1} A J_{t}^{\alpha} S_{K}(t) C x=C^{-1} S_{K}(t) C x-C^{-1} \Theta(t) C^{2} x .
$$

Since $A$ is closed, (26) indicates that

$$
J_{t}^{\alpha} S_{K}(t) C^{-1} A C x=S_{K}(t) x-C^{-1} \Theta(t) C x .
$$

Hence $x \in D(B)$ and $B x=C^{-1} A C x$. Hence (iii) holds.

Remark 5. Part of Lemma 3 and Theorem 4 are included in [40]. However, our proofs are more specific.

By the above theorem, for a nondegenerate $K$-convoluted $\alpha$-order $C$-semigroup $\left\{S_{K}(t)\right\}_{t \geq 0}$ generated by $A$, we have $A \subset$ $B$. Moreover, in the special case $C=I$, we have $A=B$.

\section{Two Characterizations of Convoluted Fractional $C$-Semigroup}

In this section we will introduce two functional equations characterizing $K$-convoluted $\alpha$-order $C$-semigroups, just like $K$-convoluted $C$-semigroups and integrated $C$-semigroups.

Lemma 6. Assume that $\left\{S_{K}(t)\right\}_{t \geq 0}$ is a strongly continuous family of bounded linear operators on Banach space $X$. Then $\{S(t)\}_{t \geq 0}$ is a K-convoluted fractional C-semigroup if and only if

$$
\begin{aligned}
& S_{K}(t) J_{s}^{\alpha} S_{K}(s)-J_{t}^{\alpha} S_{K}(t) S_{K}(s) \\
& \quad=\Theta(t) J_{s}^{\alpha} S_{K}(s) C-\Theta(s) J_{t}^{\alpha} S_{K}(t) C, \quad s, t \geq 0 .
\end{aligned}
$$

Proof

Necessity. Assume that $\left\{S_{K}(t)\right\}_{t \geq 0}$ is a $K$-convoluted $\alpha$-order $C$-semigroup generated by $A$. Let $x \in X$. Denote by $A$ the generator of $\left\{S_{K}(t)\right\}_{t \geq 0}$. By (11), it follows that

$$
\begin{array}{r}
J_{t}^{\alpha} S_{K}(t) A J_{s}^{\alpha} S_{K}(s) x=J_{t}^{\alpha} S_{K}(t) S_{K}(s) x-J_{t}^{\alpha} S_{K}(t) \Theta(s) C x, \\
x \in X .
\end{array}
$$

In (11), replacing $x$ with $J_{s}^{\alpha} S_{K}(s) x$, it follows that

$$
\begin{array}{r}
A J_{t}^{\alpha} S_{K}(t) J_{s}^{\alpha} S_{K}(s) x=S_{K}(t) J_{s}^{\alpha} S_{K}(s) x-\Theta(t) C J_{s}^{\alpha} S_{K}(s) x, \\
x \in X .
\end{array}
$$

Observe that $J_{t}^{\alpha} S_{K}(t) J_{s}^{\alpha} S_{K}(s) x \in D(A)$ and

$$
J_{t}^{\alpha} S_{K}(t) A J_{s}^{\alpha} S_{K}(s) x=A J_{t}^{\alpha} S_{K}(t) J_{s}^{\alpha} S_{K}(s) x .
$$

The combination of (28), (29), and (30) implies that

$$
\begin{array}{r}
S_{K}(t) J_{s}^{\alpha} S_{K}(s)-J_{t}^{\alpha} S_{K}(t) S_{K}(s) \\
=\Theta(t) J_{s}^{\alpha} S_{K}(s) C-\Theta(s) J_{t}^{\alpha} S_{K}(t) C, \\
s, t \geq 0 .
\end{array}
$$

Sufficiency. Assume that (27) holds. Define operator $B$ as in (17). Then $B$ is closed. Moreover, (27) implies that $J_{s}^{\alpha} S_{K}(s) x \in$ $D(B)$ and $B J_{s}^{\alpha} S_{K}(s) x=S_{K}(s) x-\Theta(s) C x$. Hence $\left\{S_{K}(t)\right\}_{t \geq 0}$ is a $K$-convoluted $\alpha$-order $C$-semigroup generated by $B=$ $C^{-1} A C$. The proof is therefore complete.

Remark 7. We have to mention that the theory of $(a, k)$ regularized $C$-resolvent families is a generalization of the theory of $(a, k)$-regularized resolvent families developed by Lizama and Poblete (see the survey paper [37]) and has been used by the same mentioned author in the development of many properties concerning the study of fractional differential equations [41]. Therefore, Lemma 6 is a natural extension to the case $C \neq I$ of the results in [37] and the proof is also motivated by that of [37].

We note that (27) does not express the functional equation in terms of the sum of time variables: $s+t$. As we describe semigroups and convoluted $C$-semigroups, this is very important in concrete applications of the functional equation modeling evolution in time. Below we will find a novel functional equation in terms of the sum of time variables $s+t$ to describe $K$-convoluted $\alpha$-order $C$-semigroup.

Theorem 8. Assume that $\left\{S_{K}(t)\right\}_{t \geq 0}$ is a K-convoluted $\alpha$-order $C$-semigroup generated by A on the Banach space $X$. Then

(i) $S_{K}(0)=0$;

(ii) $C S_{K}(\cdot)=S_{K}(\cdot) C$;

(iii) for any $t, s \geq 0$,

$$
\begin{aligned}
\alpha \int_{0}^{t} \int_{0}^{s} \frac{S_{K}(\tau) S_{K}(\sigma)}{(t+s-\sigma-\tau)^{1+\alpha}} d \tau d \sigma \\
\quad=\int_{0}^{t+s} \int_{0}^{t+s-\sigma} \frac{K(\tau) S_{K}(\sigma) C}{(t+s-\tau-\sigma)^{\alpha}} d \tau d \sigma \\
\quad-\int_{0}^{t} \int_{0}^{t+s-\sigma} \frac{K(\tau) S_{K}(\sigma) C}{(t+s-\tau-\sigma)^{\alpha}} d \tau d \sigma \\
\quad-\int_{0}^{s} \int_{0}^{t+s-\sigma} \frac{K(\tau) S_{K}(\sigma) C}{(t+s-\tau-\sigma)^{\alpha}} d \tau d \sigma,
\end{aligned}
$$

where the integrals are taken in the strong sense.

Proof. Denote by $L(t, s)$ and $R(t, s)$ the left and right hand sides of equality (32), respectively. We only have to prove that 
$L(t, s)=R(t, s)$ for all $t, s \geq 0$. For brevity, we introduce the following notations. Let

$$
\begin{gathered}
H(t, s)=S_{K}(t) J_{s}^{\alpha} S_{K}(s)-J_{t}^{\alpha} S_{K}(t) S_{K}(s), \\
K(t, s)=\Theta(t) J_{s}^{\alpha} S_{K}(s) C-\Theta(s) J_{t}^{\alpha} S_{K}(t) C, \quad t, s \geq 0 .
\end{gathered}
$$

Moreover, for sufficiently large $b>0$ denote by $g_{b}(t)$ the truncation of $S_{K}(t)$ at $b$ and by $R_{b}(t, s), L_{b}(t, s), H_{b}(t, s)$, and $K_{b}(t, s)$ the quantities obtained by replacing $S_{K}(t)$ with $g_{b}(t)$ in $R(t, s), L(t, s), H(t, s)$, and $K(t, s)$, respectively.

By $[38,(20)]$, it follows that the Laplace transform of $L_{b}(t, s)$ with respect to $t$ and $s$ is given by

$$
\widehat{L}_{b}(\mu, \lambda)=\frac{\Gamma(1-\alpha)}{\lambda-\mu}\left(\lambda^{\alpha}-\mu^{\alpha}\right) \widehat{g}_{b}(\mu) \widehat{g}_{b}(\lambda) .
$$

We now compute the Laplace transform of $R_{b}(t, s)$ with respect to $s$ and $t$. It can be shown that, for any $t \geq 0$,

$\widehat{R}_{b}(t, \lambda)$

$$
\begin{aligned}
& =\int_{0}^{\infty} e^{-\lambda s}\left[\int_{t}^{t+s} \int_{0}^{t+s-\sigma} \frac{K(\tau) g_{b}(\sigma) C}{(t+s-\tau-\sigma)^{\alpha}} d \tau d \sigma\right. \\
& \left.-\int_{0}^{s} \int_{0}^{t+s-\sigma} \frac{K(\tau) g_{b}(\sigma) C}{(t+s-\tau-\sigma)^{\alpha}} d \tau d \sigma\right] d s \\
& =\int_{t}^{\infty} g_{b}(\sigma) \int_{\sigma-t}^{\infty} \int_{0}^{t+s-\sigma} \frac{e^{-\lambda s} K(\tau) C}{(t+s-\tau-\sigma)^{\alpha}} d \tau d s d \sigma \\
& -\int_{0}^{\infty} g_{b}(\sigma) \int_{\sigma}^{\infty} \int_{0}^{t+s-\sigma} \frac{e^{-\lambda s} K(\tau) C}{(t+s-\tau-\sigma)^{\alpha}} d \tau d s d \sigma \\
& =\int_{t}^{\infty} g_{b}(\sigma) e^{\lambda(t-\sigma)} \int_{0}^{\infty} e^{-\lambda r} \int_{0}^{r}(r-\tau)^{-\alpha} K(\tau) C d \tau d r d \sigma \\
& -\int_{0}^{\infty} e^{-\lambda(t-\sigma)} g_{b}(\sigma) \int_{0}^{\infty} e^{-\lambda(t+r)} \int_{0}^{t+r} \frac{K(\tau) C}{(t+r-\tau)^{\alpha}} d \tau d r d \sigma \\
& =\int_{t}^{\infty} g_{b}(\sigma) e^{\lambda(t-\sigma)} \int_{0}^{\infty} e^{-\lambda r} \int_{0}^{r}(r-\tau)^{-\alpha} K(\tau) C d \tau d r d \sigma \\
& -\int_{0}^{\infty} e^{-\lambda(t-\sigma)} g_{b}(\sigma) \int_{t}^{\infty} e^{-\lambda r} \int_{0}^{r} \frac{K(\tau) C}{(r-\tau)^{\alpha}} d \tau d r d \sigma \\
& =\int_{0}^{\infty} g_{b}(\sigma) e^{\lambda(t-\sigma)} \int_{0}^{t} e^{-\lambda r} \int_{0}^{r}(r-\tau)^{-\alpha} K(\tau) C d \tau d r d \sigma \\
& -\int_{0}^{t} e^{-\lambda(t-\sigma)} g_{b}(\sigma) \int_{0}^{\infty} e^{-\lambda r} \int_{0}^{r} \frac{K(\tau) C}{(r-\tau)^{\alpha}} d \tau d r d \sigma \\
& =\int_{0}^{\infty} g_{b}(\sigma) e^{-\lambda \sigma} \int_{0}^{t} e^{\lambda(t-r)} \int_{0}^{r}(r-\tau)^{-\alpha} K(\tau) C d \tau d r d \sigma \\
& -\int_{0}^{t} e^{-\lambda(t-\sigma)} g_{b}(\sigma) \int_{0}^{\infty} e^{-\lambda r} \int_{0}^{r} \frac{K(\tau) C}{(r-\tau)^{\alpha}} d \tau d r d \sigma
\end{aligned}
$$

$$
\begin{aligned}
= & \widehat{g}_{b}(\lambda) \int_{0}^{t} e^{\lambda(t-r)} \int_{0}^{r}(r-\tau)^{-\alpha} K(\tau) C d \tau d r \\
& -\lambda^{\alpha-1} \Gamma(1-\alpha) \int_{0}^{t} e^{\lambda(t-\sigma)} g_{b}(\sigma) d \sigma \widehat{K}(\lambda) C,
\end{aligned}
$$

with which we can further take Laplace transform of $\widehat{R}_{b}(t, \lambda)$ with respect to $t$ as follows:

$$
\begin{aligned}
\widehat{R}_{b}(\mu, \lambda) & \int_{0}^{\infty} e^{-\mu t} \widehat{g}_{b}(\lambda) \int_{0}^{t} e^{\lambda(t-r)} \int_{0}^{r}(r-\tau)^{-\alpha} K(\tau) C d \tau d r d t \\
& -\int_{0}^{\infty} e^{-\mu t} \lambda^{\alpha-1} \Gamma(1-\alpha) \int_{0}^{t} e^{\lambda(t-\sigma)} g_{b}(\sigma) d \sigma \widehat{K}(\lambda) d t \\
= & \frac{\mu^{\alpha-1} \Gamma(1-\alpha) \widehat{g}_{b}(\lambda) \widehat{K}(\mu) C}{\mu-\lambda} \\
& -\frac{\lambda^{\alpha-1} \Gamma(1-\alpha) \widehat{g}_{b}(\mu) \widehat{K}(\lambda) C}{\mu-\lambda} .
\end{aligned}
$$

We set

$$
\begin{aligned}
& P_{b}(t, s)=\alpha \int_{0}^{t} \int_{0}^{s} \frac{H_{b}\left(r_{1}, r_{2}\right)}{\left(t+s-r_{1}-r_{2}\right)^{1+\alpha}} d r_{1} d r_{2}, \\
& Q_{b}(t, s)=\alpha \int_{0}^{t} \int_{0}^{s} \frac{K_{b}\left(r_{1}, r_{2}\right)}{\left(t+s-r_{1}-r_{2}\right)^{1+\alpha}} d r_{1} d r_{2} .
\end{aligned}
$$

By Lemma 6 , it follows that $H(t, s)=K(t, s)$ for any $t, s \geq 0$. Thus, for all $t, s \geq 0$,

$$
\lim _{b \rightarrow \infty} P_{b}(t, s)=\lim _{b \rightarrow \infty} K_{b}(t, s) .
$$

The Laplace transform of $P_{a}(t, s)$ is

$$
\widehat{P}_{b}(\mu, \lambda)=\left(\mu^{-\alpha}-\lambda^{-\alpha}\right) \widehat{L}_{b}(\mu, \lambda) .
$$

Therefore,

$\widehat{\mathrm{Q}}_{b}(t, \lambda)$

$$
\begin{aligned}
& =\alpha \int_{0}^{\infty} e^{-\lambda s} \int_{0}^{t} \int_{0}^{s} \frac{\Theta\left(r_{2}\right) J_{r_{1}}^{\alpha} g_{b}\left(r_{1}\right) C-\Theta\left(r_{1}\right) J_{r_{2}}^{\alpha} g_{b}\left(r_{2}\right) C}{\left(t+s-r_{1}-r_{2}\right)^{1+\alpha}} d r_{1} d r_{2} d s \\
& =\alpha \int_{0}^{t} \int_{0}^{\infty} e^{-\lambda s} \int_{0}^{s} \frac{\Theta\left(r_{2}\right) J_{r_{1}}^{\alpha} g_{b}\left(r_{1}\right) C-\Theta\left(r_{1}\right) J_{r_{2}}^{\alpha} g_{b}\left(r_{2}\right) C}{\left(t+s-r_{1}-r_{2}\right)^{1+\alpha}} d r_{1} d s d r_{2} \\
& =\alpha \int_{0}^{t}\left(\Theta\left(r_{2}\right) \lambda^{-\alpha} \widehat{g}_{b}(\lambda) C-\lambda^{-1} \widehat{K}(\lambda) J_{r_{2}}^{\alpha} g_{b}\left(r_{2}\right) C\right) \widehat{\Pi}\left(t-r_{2}, \lambda\right) d r_{2},
\end{aligned}
$$


where $\widehat{\Pi}(t, \lambda)$ stands for the Laplace transform of the function $(t+s)^{-\alpha-1}$ of $s$. Thus, we obtain

$$
\begin{aligned}
\widehat{Q}_{b} & (\mu, \lambda) \\
= & \alpha\left[\lambda^{-\alpha} \mu^{-1} \widehat{K}(\mu) \widehat{g}_{b}(\lambda) C-\mu^{-\alpha} \lambda^{-1} \widehat{K}(\lambda) \widehat{g}_{b}(\mu) C\right] \widehat{\Pi}(\mu, \lambda) \\
= & \alpha \lambda^{-\alpha} \mu^{-\alpha}\left(\mu^{\alpha-1} \widehat{K}(\mu) \widehat{g}_{b}(\lambda) C-\lambda^{\alpha-1} \widehat{K}(\lambda) \widehat{g}_{b}(\mu) C\right) \\
& \times \int_{0}^{\infty} e^{-\mu t} \int_{0}^{\infty} e^{-\lambda s} \frac{1}{(t+s)^{1+\alpha}} d s d t \\
= & \alpha \lambda^{-\alpha} \mu^{-\alpha}\left(\mu^{\alpha-1} \widehat{K}(\mu) \widehat{g}_{b}(\lambda) C-\lambda^{\alpha-1} \widehat{K}(\lambda) \widehat{g}_{b}(\mu) C\right) \\
& \times \frac{\Gamma(1-\alpha)}{\alpha(\lambda-\mu)}\left(\lambda^{\alpha}-\mu^{\alpha}\right) \\
= & \left(\mu^{-\alpha}-\lambda^{-\alpha}\right) \widehat{R}_{b}(\mu, \lambda) .
\end{aligned}
$$

By virtue of inverse Laplace transform, we obtain from (39) and (41) that

$$
\begin{gathered}
P_{b}(t, s)=\left(J_{s}^{\alpha}-J_{t}^{\alpha}\right) L_{b}(t, s), \\
Q_{b}(t, s)=\left(J_{s}^{\alpha}-J_{t}^{\alpha}\right) R_{b}(t, s), \quad \forall t, s \geq 0 .
\end{gathered}
$$

The combination of (38) and (42) implies that

$$
\left(J_{s}^{\alpha}-J_{t}^{\alpha}\right) R(t, s)=\left(J_{s}^{\alpha}-J_{t}^{\alpha}\right) L(t, s), \quad \forall t, s \geq 0 .
$$

Therefore, $R(t, s)=L(t, s)$, for all $t, s \geq 0$. This completes the proof.

Theorem 9. Assume that $\left\{S_{K}(t)\right\}_{t \geq 0}$ is a strongly continuous nondegenerate operator family on Banach space X. Suppose that the conditions (i), (ii), and (iii) in Theorem 8 hold. Then $\left\{S_{K}(t)\right\}_{t \geq 0}$ is a nondegenerate $K$-convoluted $\alpha$-order $C$ semigroup generated by $B$, where $B$ is defined as in Theorem 4.

Proof. Obviously, the right hand side of (32) is symmetric with respect to $t$ and $s$. Hence the left hand side is symmetric with respect to $t$ and $s$. By the proof of Proposition 1 of [38], it follows that $\left\{S_{K}(t)\right\}_{t \geq 0}$ is commutative; that is,

$$
S_{K}(t) S_{K}(s)=S_{K}(s) S_{K}(t), \quad \forall t, s \geq 0 .
$$

For any $x \in D(B)$, there holds

$$
S_{K}(s) x-\Theta(s) C x=J_{s}^{\alpha} S_{K}(s) B x, \quad s \geq 0 .
$$

Applying $S_{k}(t)$ on both sides of (45) and using the commutativity $(44)$ of $\left\{S_{K}(s)\right\}_{t \geq 0}$, we obtain

$$
S_{K}(s) S_{K}(t) x-\Theta(s) C S_{K}(t) x=J_{s}^{\alpha} S_{K}(s) S_{K}(t) B x, \quad s \geq 0 .
$$

This implies by definition that $S_{K}(t) x \in D(B)$ and $B S_{K}(t) x=$ $S_{K}(t) B x$.

Denote by $L(t, s)$ and $R(t, s)$ the left and right hand sides of equality (32), respectively. For any $c \geq 0$, denote by $g_{c}(t)$ the truncation of $S_{K}(t)$ at $c$; that is, $g_{c}(\sigma)=S_{K}(\sigma), 0 \leq \sigma \leq c$ and $g_{c}(\sigma)=0$ otherwise. Denote by $R_{c}(t, s)$ and $L_{c}(t, s)$ the quantities obtained by replacing $S_{K}(t)$ with $g_{c}(t)$ in $R(t, s)$, $L(t, s)$, respectively.

Let $x \in X$ and $t \geq 0$ be fixed. Define the function $H_{t}(r, s)$ for $r, s \geq 0$ by

$$
H_{t}(r, s)=\left(g_{t}(r)-\Theta(r) C\right) J_{s}^{\alpha} g_{t}(s) x .
$$

Obviously, for $0 \leq r \leq t$,

$$
H_{t}(r, t)=\left(S_{K}(r)-\Theta(r) C\right) J_{t}^{\alpha} S_{K}(t) x .
$$

Taking the Laplace transform with respect to $r$ and $s$ successively for both sides of (47), we derive

$$
\widehat{H}_{t}(\mu, \lambda)=\lambda^{-\alpha} \widehat{g}_{t}(\mu) \widehat{g}_{t}(\lambda) x-\lambda^{-\alpha} \mu^{-1} \widehat{K}(\mu) \widehat{g}_{t}(\lambda) x .
$$

The combination of (43) and (49) implies that

$$
\begin{aligned}
\widehat{H}_{t}(\mu, \lambda)= & \mu^{-\alpha} \widehat{g}_{t}(\mu) \widehat{g}_{t}(\lambda) x-\mu^{-\alpha} \lambda^{-1} \widehat{K}(\lambda) \widehat{g}_{t}(\mu) x \\
& +\frac{\lambda^{-\alpha} \mu^{-\alpha}(\lambda-\mu)}{\Gamma(1-\alpha)}\left(\widehat{R}_{t}(\mu, \lambda)-\widehat{L}_{t}(\mu, \lambda)\right) x .
\end{aligned}
$$

By virtue of inverse Laplace transform, it follows that

$$
\begin{aligned}
& H_{t}(r, s) \\
& =\left(g_{t}(s)-\Theta(s) C\right) J_{r}^{\alpha} g_{t}(r) x \\
& \quad+\frac{\left[\left({ }^{C} D_{s}^{1-\alpha}\right) J_{r}^{\alpha}-\left({ }^{C} D_{r}^{1-\alpha}\right) J_{s}^{\alpha}\right] \cdot\left[R_{t}(r, s)-L_{t}(r, s)\right] x}{\Gamma(1-\alpha)} .
\end{aligned}
$$

Here the Laplace transform formula

$$
\begin{gathered}
\widehat{{ }^{C} D_{t}^{\beta} f}(\lambda)=\lambda^{\beta} \widehat{f}(\lambda)-\lambda^{\beta-1} f(0), \\
0<\beta<1, f \in C([0, \infty), X),
\end{gathered}
$$

is used.

By the definition of $g_{t}$, it follows that $L_{t}(r, s)=R_{t}(r, s)$ for all $0 \leq s, r \leq t$; we have that

$$
\begin{array}{r}
H_{t}(r, s)=\left(S_{K}(s)-\Theta(s) C\right) J_{r}^{\alpha} S_{K}(r) x, \\
\forall 0 \leq r, s \leq t .
\end{array}
$$

In particular, we obtain

$$
\begin{array}{r}
H_{t}(r, t)=\left(S_{K}(t)-\Theta(t) C\right) J_{r}^{\alpha} S_{K}(r) x, \\
\forall 0 \leq r \leq t .
\end{array}
$$

Combining (48) and (54), we derive that

$$
\begin{aligned}
\left(S_{K}(r)-\Theta(r) C\right) J_{t}^{\alpha} S_{K}(t) x & =\left(S_{K}(t)-\Theta(t) C\right) J_{r}^{\alpha} S_{K}(r) x \\
& =J_{r}^{\alpha} S_{K}(r)\left(S_{K}(t) x-\Theta(t) C x\right) .
\end{aligned}
$$

This implies by definition that $J_{r}^{\alpha} S_{K}(r) x \in D(B)$ and

$$
B J_{r}^{\alpha} S_{K}(r) x=S_{K}(r) x-\Theta(r) C x .
$$

Therefore, $\left\{S_{K}(t)\right\}_{t \geq 0}$ is a $K$-convoluted $\alpha$-order $C$-semigroup generated by $B$. 
The combination of Theorems 8 and 9 indicates the following theorem.

Theorem 10. Assume that $\left\{S_{K}(t)\right\}_{t \geq 0}$ is a strongly continuous operator family on Banach space $X$. Then $\left\{S_{K}(t)\right\}_{t \geq 0}$ is a $K$ convoluted $\alpha$-order C-semigroup if and only if (i), (ii), and (iii) in Theorem 8 hold.

Remark 11. Letting $\alpha \rightarrow 1^{-}$, (32) becomes the $K$-convoluted $C$-semigroup defined in [1]. Indeed, if for each $x$ in some dense set the mapping $t \mapsto S_{K}(t) x$ is continuously differentiable in $[0, \infty)$, then the limits of two sides of the equality (32) multiplied with $1-\alpha$ equal $S_{K}(t) S_{K}(s) x$ and $\left[\int_{0}^{t+s}-\int_{0}^{t}-\int_{0}^{s}\right] K(t+s-r) S_{K}(r) C x d r$, respectively. The combination of the above fact and Theorem 10 implies that $K$ convoluted $\alpha$-order $C$-semigroup can be deemed as a natural extension of $K$-convoluted $C$-semigroup.

\section{Fractional Abstract Cauchy Problems}

In this section we will apply the theory of $K$-convoluted $\alpha$-order $C$-semigroups developed in Sections 2 and 3 to fractional abstract Cauchy problems (FACP). We begin with the definition of classical solution of FACP.

Definition 12. A function $u \in C([0, \infty), X)$ is called a classical solution of FACP, if $u(0)=0, u \in C([0, \infty), D(A))$, and the mapping $t \mapsto \int_{0}^{t}(t-s)^{-\alpha} u(s) d s$ is continuously differentiable such that $(1 / \Gamma(1-\alpha))(d / d t) \int_{0}^{t}(t-\sigma)^{-\alpha} u(\sigma) d \sigma=A u(t)+$ $\Theta(t) C x, t \geq 0$ holds.

Remark 13. In Definition 12, $(1 / \Gamma(1-\alpha))(d / d t) \int_{0}^{t}(t-$ $\sigma)^{-\alpha} u(\sigma)$ is just the modified Caputo derivative of $u(t)$ because $u(0)=0$.

Definition 14. The fractional abstract Cauchy problem (FACP) is said to be $C$-well-posed if for any $x \in X$, there exists a unique solution $u(t, x)$, and $X \ni x_{n} \rightarrow 0$ implies that $u\left(t, x_{n}\right) \rightarrow 0$ as $n \rightarrow \infty$ in $X$, uniformly on any compact subinterval of $[0, \infty)$.

Theorem 15. Assume that $A$ is closed, $C A \subset A C$, and FACP is $C$-well-posed. Then A generates a $K$-convoluted $\alpha$-order $C$ semigroup.

Proof. For any $x \in X$, denote by $u(t, x), t \geq 0$, the solution of FACP. Denote by $S(t) x={ }^{C} D_{t}^{\alpha} u(t, x), x \in X$. Obviously, $S(0)={ }^{C} D_{t}^{\alpha} u(0, \cdot)=0$ and $\{S(t)\}_{t \geq 0}$ is strongly continuous. Since $C A \subset A C$, we have

$$
\begin{aligned}
{ }^{C} D_{t}^{\alpha}[C u(t, x)] & =C^{C} D_{t}^{\alpha} u(t, x) \\
& =C A u(t, x)+\Theta(t) C^{2} x \\
& =A[C u(t, x)]+\Theta(t) C(C x) .
\end{aligned}
$$

By the uniqueness of the solution of ACP, we have $C u(t, x)=$ $u(t, C x)$. Then

$$
\begin{aligned}
C S(t) x & ={ }^{C} D_{t}^{\alpha} C u(t, x)={ }^{C} D_{t}^{\alpha} u(t, C x) \\
& =S(t) C x, \quad t \geq 0 .
\end{aligned}
$$

By (1.21) of [27], it follows that

$$
J_{t}^{\alpha} S(t) x=J_{t}^{\alpha C} D_{t}^{\alpha} u(t, x)=u(t, x)-u(0, x)=u(t, x) .
$$

Then $J_{t}^{\alpha} S(t) x \in D(A)$ and

$$
A J_{t}^{\alpha} S(t) x+\Theta(t) C x=A u(t, x)+\Theta(t) C x=S(t) x .
$$

Below we will show that $S(t)$ is continuous, a.e. $S(t) \in$ $B(X), t \in[0, \infty)$. By (59), it follows that $V(t) x=: J_{t}^{\alpha} S(t) x$ is the solution of ACP.

Let $y \in D(A)$. Define $\widetilde{S}(t)=J_{t}^{\alpha} S(t) A y+\Theta(t) C y$. Then $J_{t}^{\alpha} \widetilde{S}(t)=J_{t}^{\alpha} J_{t}^{\alpha} S(t) A y+J_{t}^{\alpha} \Theta(t) C y$. Since $J_{t}^{\alpha} S(t) A y \in D(A)$, $C y \in D(A)$, and $A$ is closed, it follows that $J_{t}^{\alpha} \widetilde{S}(t) \in D(A)$ and

$$
\begin{aligned}
A J_{t}^{\alpha} \widetilde{S}(t) & =J_{t}^{\alpha} A J_{t}^{\alpha} S(t) A y+J_{t}^{\alpha} \Theta(t) C A y \\
& =J_{t}^{\alpha}\left(A J_{t}^{\alpha} S(t) A y+\Theta(t) C A y\right) \\
& =J_{t}^{\alpha} S(t) A y=\widetilde{S}(t)-\Theta(t) C y .
\end{aligned}
$$

Then $\widetilde{S}(t)=S(t)$ and $S(t)=J_{t}^{\alpha} S(t) A y+\Theta(t) C y$. Hence $A J_{t}^{\alpha} S(t) y=J_{t}^{\alpha} S(t) A y, y \in D(A)$. Since $A$ is closed, we have $J_{t}^{\alpha} S(t) A y=A J_{t}^{\alpha} S(t) y=J_{t}^{\alpha} A S(t) y$. By Titchmarsh's theorem, $S(t) A y=A S(t) y, t \geq 0$. The proof is complete.

Theorem 16. Assume that A generates a nondegenerate $K$ convoluted $\alpha$-order C-semigroup. Then, FACP is C-well-posed.

Proof. Since $\{S(t)\}_{t \geq 0}$ is a nondegenerate strongly continuous family generated by $A$, for any $x \in X$, we have $J^{\alpha} S(\cdot) x \in$ $C([0, \infty), D(A))$, the mapping

$$
t \longmapsto \int_{0}^{t}(t-s)^{-\alpha} J_{s}^{\alpha} S(s) d s=\Gamma(1-\alpha) \int_{0}^{t} S(t) x
$$

is continuously differentiable, and there holds

$$
\begin{aligned}
D_{t}^{\alpha} J_{t}^{\alpha} S(t) x & =\frac{d}{d t} \frac{1}{\Gamma(1-\alpha)} \int_{0}^{t}(t-s)^{-\alpha} J_{s}^{\alpha} S(s) d s \\
& =S(t) x=A J_{t}^{\alpha} S(t) x+\Theta(t) C x .
\end{aligned}
$$

Therefore, $u(t, x):=J_{t}^{\alpha} S(t) x$ is a solution of FACP.

Now we prove the uniqueness of solution of FACP. Assume that $v(t, x)$ is another solution of FACP. Then

$$
{ }^{C} D_{t}^{\alpha} v(t, x)=A v(t, x)+\Theta(t) C x, \quad t \geq 0 .
$$

Taking $\alpha$-time integrals, we derive that

$$
\begin{array}{r}
v(t, x)=J_{t}^{\alpha C} D_{t}^{\alpha} v(t, x)=J_{t}^{\alpha} A v(t, x)+J_{t}^{\alpha} \Theta(t) C x, \\
t \geq 0,
\end{array}
$$


which implies that

$$
\begin{aligned}
\Theta(t) C * v(t, x) & =\left[S(t)-A J_{t}^{\alpha} * S(t)\right] * v(t, x) \\
& =S(t) * v(t, x)-A J_{t}^{\alpha} * S(t) * v(t, x), \\
& t \geq 0 .
\end{aligned}
$$

Observe that $A$ is closed and communicative with $S(t)$, and $C$ is communicative with $S(t)$. By (67), it follows that

$$
\begin{aligned}
\Theta(t) * C v(t, x) & =\Theta(t) C * v(t, x) \\
& =S(t) * v(t, x)-S(t) * J_{t}^{\alpha} * A v(t, x) \\
& =S(t) *\left[v(t, x)-J_{t}^{\alpha} * A v(t, x)\right] \\
& =S(t) * J_{t}^{\alpha} \Theta(t) C x \\
& =\Theta(t) C * J_{t}^{\alpha} S(t) x \\
& =\Theta(t) * C J_{t}^{\alpha} S(t) x, \quad t \geq 0 .
\end{aligned}
$$

Since $S$ is nondegenerate, it follows that $C v(t, x)=C J_{t}^{\alpha} S(t) x$, $t \geq 0$. Then $v(t, x)=J_{t}^{\alpha} S(t) x, t \geq 0$ because $C$ is injective.

For any $T>0$, let $X \ni x_{n} \rightarrow 0$; we have

$$
\begin{aligned}
\left\|u\left(t, x_{n}\right)\right\| & =\left\|J_{t}^{\alpha} S(t) x_{n}\right\| \\
& \leq \frac{T^{\alpha} \sup _{t \geq T}\|S(t)\|}{\Gamma(\alpha+1)}\left\|x_{n}\right\|, \quad t \in[0, T],
\end{aligned}
$$

which implies that $u\left(t, x_{n}\right)$ tends to 0 uniformly on $t \in[0, T]$. This completes the proof.

The combination of Lemma 3 and Theorems 15 and 16 implies the following theorem.

Theorem 17. The operator $A$ generates a K-convoluted $\alpha$ order $C$-semigroup if and only if $A$ is closed, $C A \subset A C$, and FACP is C-well-posed.

Corollary 18. Assume that there exists a nondegenerate strongly continuous operator family $\{S(t)\}_{t \geq 0}$ on Banach space $X$ such that (27) holds, and B defined in Theorem 4 is equal to $A$. Then FACP is C-well-posed.

Corollary 19. Assume that there exists a nondegnerate strongly continuous operator family $\{S(t)\}_{t \geq 0}$ on Banach space $X$ satisfying ( $i$ ), (ii), and (iii) in Theorem 8 , and $B$ defined in Theorem 4 is equal to $A$. Then FACP is C-well-posed.

Corollary 20. Assume that $\{S(t)\}_{t \geq 0}$ is a nondegnerate strongly continuous operator family on the Banach space $X$. Suppose that $A=C^{-1} A C$. Then the following statements are equivalent:

(1) $\{S(t)\}_{t \geq 0}$ is a K-convoluted $\alpha$-order C-semigroup generated by $A$;

(2) $A$ is a closed linear operator on $X$, and FACP is C-wellposed;
(3) $\{S(t)\}_{t \geq 0}$ satisfies (27), and $B=A$, where $B$ is defined as in Theorem 4;

(4) $\{S(t)\}_{t \geq 0}$ satisfies (i), (ii), and (iii) in Theorem 8 , and $B=A$, where $B$ is defined as in Theorem 4 .

\section{Conflict of Interests}

The authors declare that there is no conflict of interests regarding the publication of this paper.

\section{Acknowledgments}

This work was supported by NSF of China (Grant nos. 11301412, 11131006, 41390450, and 11271297), Research Fund for the Doctoral Program of Higher Education of China (Grant no. 20130201120053), the Fundamental Research Funds for the Central Universities (Grant no. 2012jdhz52), and the National Basic Research Program of China (Grant no. 2013CB329404).

\section{References}

[1] M. Kostić, "Convoluted $C$-cosine functions and convoluted C-semigroups," Bulletin, Classe des Sciences Mathématiques et Naturelles, Sciences Mathématiques, vol. 127, no. 28, pp. 75-92, 2003.

[2] W. Arendt, "Vector-valued Laplace transforms and Cauchy problems," Israel Journal of Mathematics, vol. 59, no. 3, pp. 327352, 1987.

[3] W. Arendt, O. El-Mennaoui, and V. Kéyantuo, "Local integrated semigroups: evolution with jumps of regularity," Journal of Mathematical Analysis and Applications, vol. 186, no. 2, pp. 572595, 1994.

[4] W. Arendt, C. J. K. Batty, M. Hieber, and F. Neubrander, VectorValued Laplace Transforms and Cauchy Problems, Birkhäuser, Basel, Switzerland, 2001.

[5] H. Kellerman and M. Hieber, "Integrated semigroups," Journal of Functional Analysis, vol. 84, no. 1, pp. 160-180, 1989.

[6] F. Neubrander, "Integrated semigroups and their applications to the abstract Cauchy problem," Pacific Journal of Mathematics, vol. 135, pp. 233-251, 1989.

[7] M. Hieber, "Laplace transforms and $\alpha$-times integrated semigroups," Forum Mathematicum, vol. 3, no. 6, pp. 595-612, 1991.

[8] P. J. Miana, " $\alpha$-times integrated semigroups and fractional derivation," Forum Mathematicum, vol. 14, no. 1, pp. 23-46, 2002.

[9] M. Mijatović, S. Pilipović, and F. Vajzović, " $\alpha$-times integrated semigroups $\left(\alpha \in R^{+}\right.$)," Journal of Mathematical Analysis and Applications, vol. 210, no. 2, pp. 790-803, 1997.

[10] Y. C. Li, Integral C-semigroups and C-cosine functions of operators on locally convex spaces [Ph.D. dissertation], National Central University, 1991.

[11] Y. C. Li and S. Y. Shaw, "On generators of integrated Csemigroups and $C$-cosine functions," Semigroup Forum, vol. 47, no. 1, pp. 29-35, 1993.

[12] Y. C. Li and S. Y. Shaw, " $N$-times integrated $C$-semigroups and the abstract Cauchy problem," Taiwanese Journal of Mathematics, vol. 1, no. 1, pp. 75-102, 1997. 
[13] C. C. Kuo and S. Y. Shaw, "On $\alpha$-times integrated $C$-semigroups and the abstract Cauchy problem," Studia Mathematica, vol. 142, no. 3, pp. 201-217, 2000.

[14] I. Cioranescu, "Local convoluted semigroups," in Evolution Equations, pp. 107-122, Dekker, New York, NY, USA, 1995.

[15] I. Cioranescu and G. Lumer, "Problèmes d'évolution régularisés par un noyau général $K(t)$. Formule de Duhamel, prolongements, théorèmes de génération," Comptes Rendus de l'Académie des Sciences. Série I, vol. 319, no. 12, pp. 1273-1278, 1994.

[16] I. Cioranescu and G. Lumer, "On $K(t)$-convoluted semigroups," in Recent Developments in Evolution Equations, pp. 86-93, Longman Scientific \& Technical, Harlow, UK, 1995.

[17] P. C. Kunstmann, "Stationary dense operators and generation of non-dense distribution semigroups," Journal of Operator Theory, vol. 37, no. 1, pp. 111-120, 1997.

[18] I. V. Melnikova and A. Filinkov, Abstract Cauchy Problems: Three Approaches, Chapman \& Hall/CRC, Boca Raton, Fla, USA, 2001.

[19] V. Keyantuo, C. Müller, and P. Vieten, "The Hille-Yosida theorem for local convoluted semigroups," Proceedings of the Edinburgh Mathematical Society, vol. 46, no. 2, pp. 395-413, 2003.

[20] M. Kostić and S. Pilipović, "Global convoluted semigroups," Mathematische Nachrichten, vol. 280, no. 15, pp. 1727-1743, 2007.

[21] M. Kostić and S. Pilipović, "Convoluted C-cosine functions and semigroups. Relations with ultradistribution and hyperfunction sines," Journal of Mathematical Analysis and Applications, vol. 338, no. 2, pp. 1224-1242, 2008.

[22] S. D. Eidelman and A. N. Kochubei, "Cauchy problem for fractional diffusion equations," Journal of Differential Equations, vol. 199, no. 2, pp. 211-255, 2004.

[23] V. Lakshmikanthan and S. Leela, Theory of Fractional Dynamic Systems, Cambridge Academic Publishers, Cambridge, UK, 2009.

[24] M. M. Meerschaert, E. Nane, and P. Vellaisamy, "Fractional Cauchy problems on bounded domains," The Annals of Probability, vol. 37, no. 3, pp. 979-1007, 2009.

[25] R. Metzler and J. Klafter, "The random walk's guide to anomalous diffusion: a fractional dynamics approach," Physics Reports, vol. 339, no. 1, pp. 1-77, 2000.

[26] I. Podlubny, Fractional Differential Equations, Academic Press, New York, NY, USA, 1999.

[27] E. G. Bajlekova, Fractional Evolution Equations in Banach Spaces, University Press Facilities, Eindhoven University of Technology, Eindhoven, The Netherlands, 2001.

[28] G. da Prato and M. Iannelli, "Linear integro-differential equations in Banach spaces," Rendiconti del Seminario Matematico dell'Università di Padova, vol. 62, pp. 207-219, 1980.

[29] M. Li, Q. Zheng, and J. Zhang, "Regularized resolvent families," Taiwanese Journal of Mathematics, vol. 11, no. 1, pp. 117-133, 2007.

[30] C. Lizama, "Regularized solutions for abstract Volterra equations," Journal of Mathematical Analysis and Applications, vol. 243, no. 2, pp. 278-292, 2000.

[31] J. Prüss, Evolutionary Integral Equations and Applications, Birkhäuser, Basel, Switzerland, 1993.

[32] C. Chen and M. Li, "On fractional resolvent operator functions," Semigroup Forum, vol. 80, no. 1, pp. 121-142, 2010.

[33] L. Kexue and P. Jigen, "Fractional abstract Cauchy problems," Integral Equations and Operator Theory, vol. 70, no. 3, pp. 333361, 2011.
[34] L. Kexue and P. Jigen, "Fractional resolvents and fractional evolution equations," Applied Mathematics Letters, vol. 25, no. 5, pp. 808-812, 2012.

[35] K. X. Li, J. G. Peng, and J. X. Jia, "Cauchy problems for fractional differential equations with Riemann-Liouville fractional derivatives," Journal of Functional Analysis, vol. 263, no. 2, pp. 476-510, 2012.

[36] Z. D. Mei, J. G. Peng, and Y. Zhang, "On general fractional abstract Cauchy problem," Communications on Pure and Applied Analysis, vol. 12, no. 6, pp. 2753-2772, 2013.

[37] C. Lizama and F. Poblete, "On a functional equation associated with $(a, k)$-regularized resolvent families," Abstract and Applied Analysis, vol. 2012, Article ID 495487, 23 pages, 2012.

[38] J. Peng and K. Li, "A novel characteristic of solution operator for the fractional abstract Cauchy problem," Journal of Mathematical Analysis and Applications, vol. 385, no. 2, pp. 786-796, 2012.

[39] Z. D. Mei, J. G. Peng, and Y. Zhang, "A characteristic of fractional resolvents," Fractional Calculus and Applied Analysis, vol. 16, no. 4, pp. 777-790, 2013.

[40] M. Kostić, “ $(a, k)$-regularized $C$-resolvent families: regularity and local properties," Abstract and Applied Analysis, vol. 2009, Article ID 858242, 27 pages, 2009.

[41] C. Lizama and G. M. N'Guerekata, "Mild solutions for abstract fractional differential equations," Applicable Analysis, vol. 92, no. 8, pp. 1731-1754, 2013. 


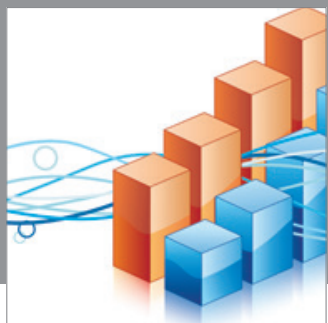

Advances in

Operations Research

mansans

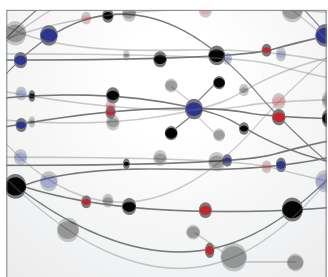

The Scientific World Journal
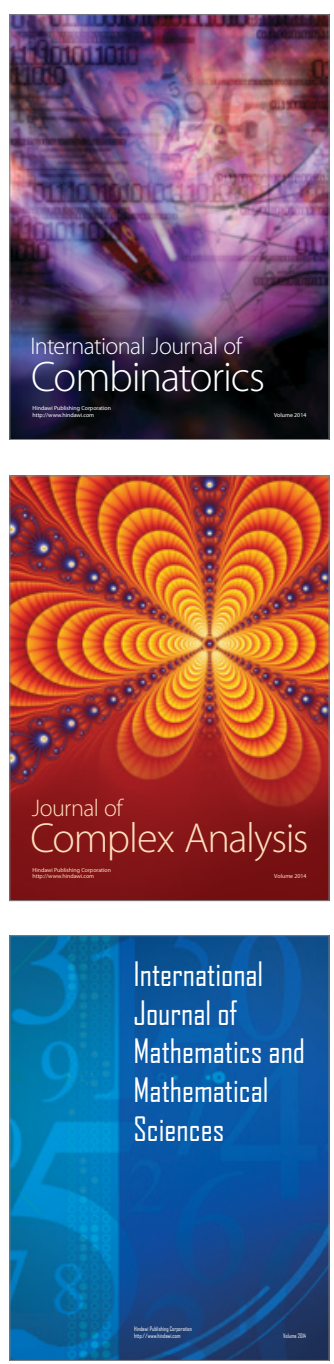
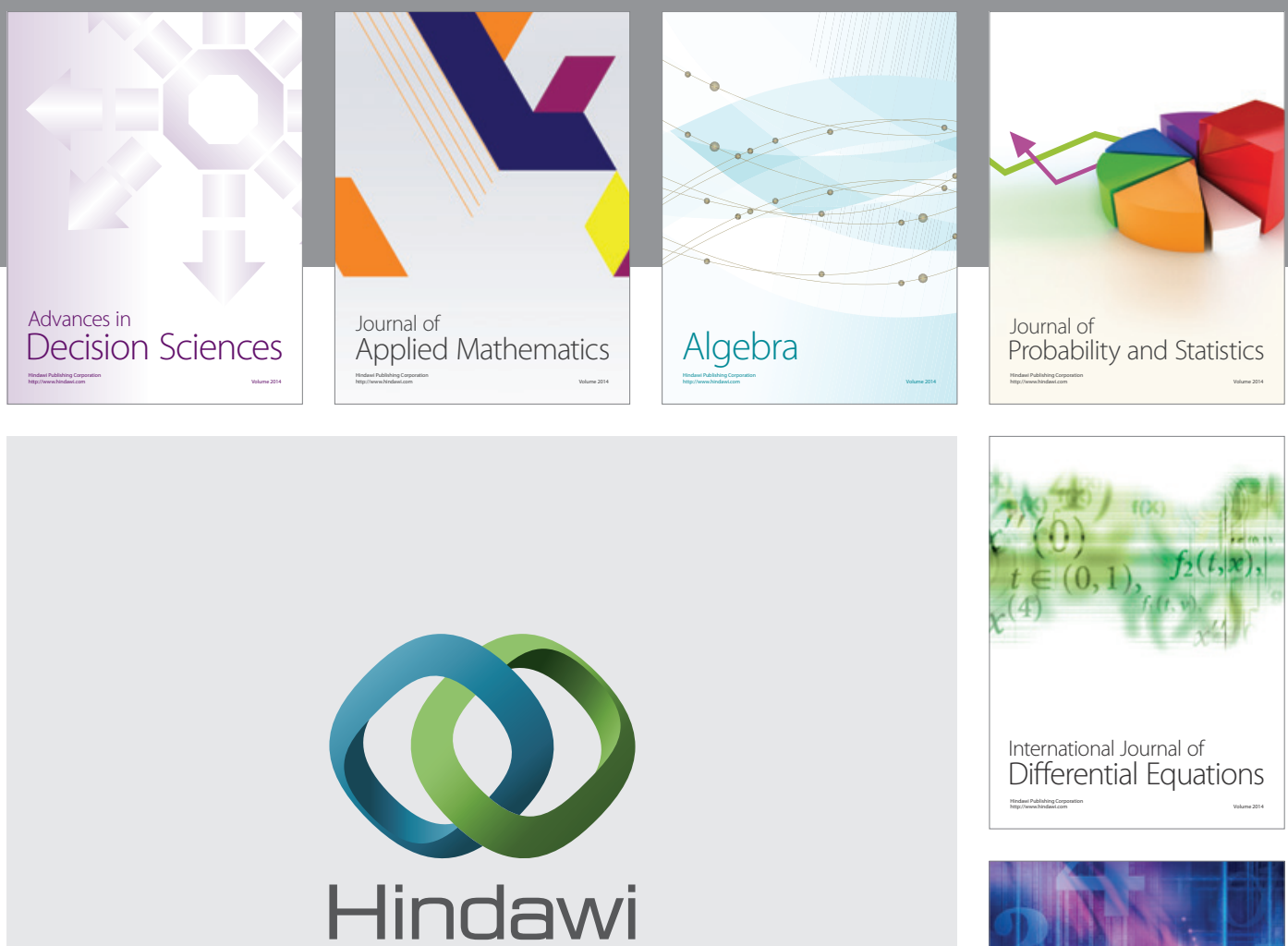

Submit your manuscripts at http://www.hindawi.com
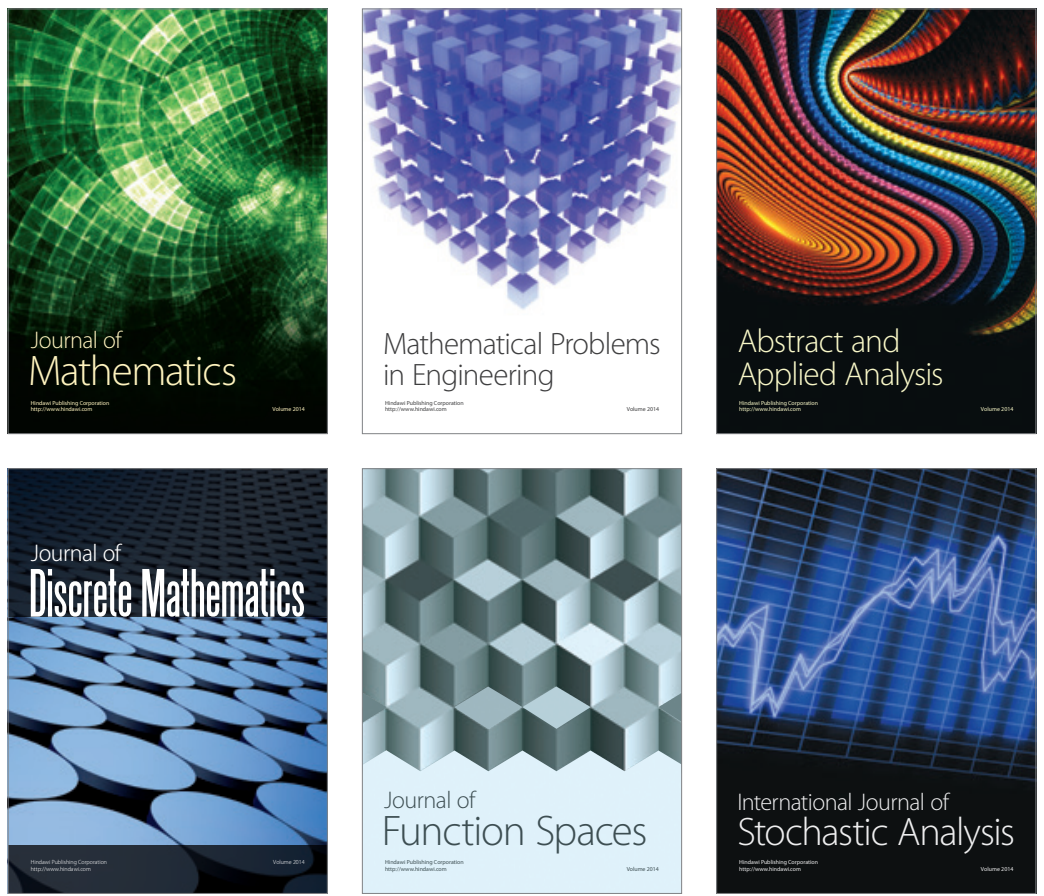

Journal of

Function Spaces

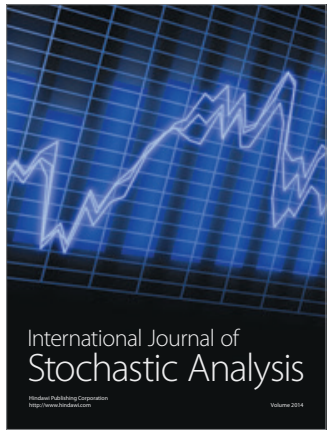

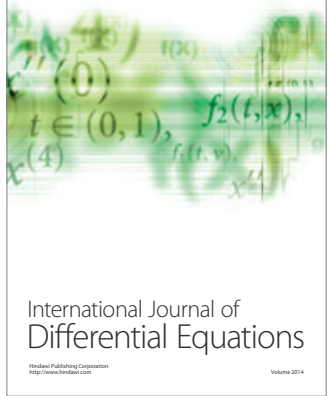
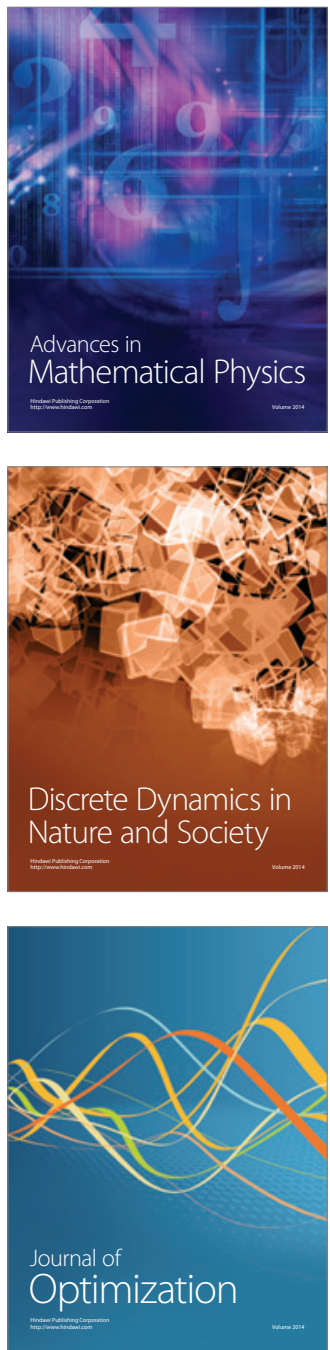